\title{
Colonoscopia com Polipectomia: Análise Crítica de Fatores de Risco e Complicações
}

\section{Colonic Snare Polipectomy: Critical Analysis of Risk Factors and Complications}

\author{
EDSON JURADO DA SILVA ${ }^{12} ;$ ALEXANDRE PELOSI²; GLÁUCIAFREITAS $^{2} ;$ ELEODORO ALMEIDA $^{1}$
}

${ }^{1}$ Serviço de Coloproctologia do Hospital dos Servidores do Estado-RJ;

${ }^{2}$ Serviço de Endoscopia Digestiva da Casa de Portugal-RJ.

\begin{abstract}
SILVA EJ; PELOSI A; FREITAS G; ALMEIDA E. Colonoscopia com Polipectomia: Análise Crítica de Fatores de Risco e Complicações. Rev bras Coloproct, 2009;29(4): 451-457.

RESUMO: Objetivo: Analisar complicações de polipectomia com alça ditérmica em cólon. Pacientes e Métodos: Estudo retrospectivo de polipectomias em colonoscopias realizadas em dois hospitais de 2001 a 2007. Teste t de Student foi usado para média, desvio padrão e qui-quadrado para números absolutos. $P$ < que 0,05 foi considerado significativo. Resultados: Foram 1687 polipectomias em 8447 colonoscopias. Sangramento imediato em $24(11,8 \%)$ em pólipos maiores do que 2 cm contra $1(0,07 \%)$ em menores p<0,01. Somente 1, maior que $2 \mathrm{~cm},(0,49 \%)$ necessitou de cirurgia para controle do sangramento p<0,01. Sangramento tardio em 7 (3,4\%), todos maiores que $2 \mathrm{~cm}$ p $<0,01$. Perfuração em 6 (2.9\%), todas em cólon direito. Não houve necessidade de cirurgia. A idade foi $59,8 \pm 6,7$ para sangramento imediato, $60 \pm 9,8$ para tardio e $63,8 \pm 16,3$ para os que não sangraram p>0,05. Síndrome pós-polipectomia em $6(0,35 \%)$. Ressecção fatiada somente em maiores que $2 \mathrm{~cm}, 89 / 116$ (77\%) sésseis e 11/87 (13\%) pediculados p<0,01. Carcinoma invasivo em 40 adenomas maiores que $2 \mathrm{~cm}(19,7 \%)$. Conclusão: Polipectomia com alça é segura, sangramento a complicação mais comum, relacionada ao tamanho da base. Perfuração vem a seguir. Ambas tem tratamento endoscópico prioritário.
\end{abstract}

Descritores: Cólon, colonoscopia, pólipo, polipectomia, complicação.

\section{INTRODUÇÃO}

Adenoma é precursor do câncer colorretal ${ }^{1-2}$ e a sua remoção serve como prevenção ${ }^{3,4,5,6}$. A intenção deste estudo é de avaliar os efeitos adversos da polipectomia realizada com alça diatérmica.

\section{PACIENTES E MÉTODOS}

Analisamos de forma retrospectiva 1687 polipectomias com alça diatérmica realizadas em 8447 colonoscopias em duas instituições médicas no período de 2001 a 2007. Duzentos e três pólipos eram maiores do que $2 \mathrm{~cm}$ de diâmetro. Teste $t$ de Student foi utilizado para análise de média e desvio padrão e qui-quadrado para números absolutos. $\mathrm{P}<$ que 0,05 foi considerado significante. As grandes lesões sésseis foram abordadas por técnica combinada, isto é, polipectomia convencional com alça diatérmica complementada por mucosectomia para retirada completa e segura das margens. Não abordamos neste estudo ressecções de lesões planas ou plana-elevadas por mucosectomia.

\section{RESULTADOS}

Em 1687 polipectomias, 203 foram para pólipos maiores do que $2 \mathrm{~cm}$, considerados grandes, sendo que o maior deles tinha $10 \mathrm{~cm}$ de diâmetro.

A tabela 1 mostra a incidência de sangramento imediato quando comparamos o tamanho dos pólipos.

Sangramento tardio, aquele que ocorreu após $24 \mathrm{~h}$ do procedimento pode ser visto na tabela 2 .

Tratalho realizado no Serviço de Coloproctologia do HSE-RJ e no Serviço de Endoscopia Digestiva da Casa de Portugal - RJ. 
Tabela 1 - Sangramento imediato pós polipectomia com alça diatérmica.

\begin{tabular}{|c|c|c|c|c|}
\hline & \multicolumn{4}{|c|}{ Tamanho do Pólipo } \\
\hline & \multicolumn{2}{|c|}{ Maior que 2 até $10 \mathrm{~cm}$} & \multicolumn{2}{|c|}{ Menor do que $1,9 \mathrm{~cm}$} \\
\hline & $\mathbf{N}$ & $\%$ & $\mathbf{N}$ & $\%$ \\
\hline Sangramento imediato & 24 & 11,8 & 1 & 0,07 \\
\hline
\end{tabular}

$P<0,01$.

Tabela 2 - Sangramento tardio.

\begin{tabular}{lccccc}
\hline & \multicolumn{4}{c}{ Tamanho do Pólipo } \\
\cline { 2 - 6 } & \multicolumn{3}{c}{ Maior que $\mathbf{2}$ até $\mathbf{1 0} \mathbf{~ c m}$} & \multicolumn{2}{c}{ Menor do que $\mathbf{1 , 9} \mathbf{~ c m}$} \\
\cline { 2 - 6 } & $\mathbf{N}$ & $\mathbf{\%}$ & $\mathbf{N}$ & $\mathbf{\%}$ \\
\hline Sangramento tardio & 7 & 3,4 & 0 & 0 \\
\hline
\end{tabular}

$P<0,01$.

Somente um paciente que sangrou necessitou de tratamento cirúrgico, todos os demais, quer tenham tido sangramento imediato ou tardio, tiveram tratamento endoscópico eficaz e o único operado tinha pólipo maior do que $2 \mathrm{~cm}$ de diâmetro.

Em 1687 polipectomias com alça diatérmica, ocorreram perfuração em 6 , todas em pólipos localizados em ceco e cólon ascendente, sendo o perfil dos pólipos descritos na tabela 3 . Todas as perfurações tiveram conduta conservadora.

Síndrome pós polipectomia também ocorreu em pólipo grande e é apresentada na tabela 4.

Nos pólipos maiores do que $2 \mathrm{~cm}$ de diâmetro a polipectomia fatiada, ou em piecemeal, foi mais freqüente nos sésseis do que nos pediculados, e isto pode ser visto na Tabela 4. Os pólipos menores do que $2 \mathrm{~cm}$ tiveram polipectomia em peça única.

As idades dos pacientes que apresentaram sangramento pós polipectomia estão apresentadas na tabela 5.

Tabela 3 - Perfuração durante polipectomia.
Complicação inusitada ocorreu em uma paciente $(0,05 \%)$, correspondendo a fratura não percebida da alça de polipectomia, acarretando fixação da cesta, basket, no pólipo ainda fixo, contornado facilmente com o uso de um segundo aparelho com corte do pedículo íntegro com auxílio do papilótomo de Huibregtse, introduzido pelo canal de instrumentação do segundo endoscópio, na realidade um esofagogastroduodenoscópio.

A presença de adenocarcinoma invasivo em adenoma ocorreu em 40 pólipos (19,7\%), todos maiores que $2 \mathrm{~cm}$ de diâmetro.

\section{DISCUSSÃO}

Os pólipos de cólon provenientes da mucosa, podem ser classificados quanto ao seu aspecto macroscópico em pediculados e sésseis e em função do tamanho em grandes, maiores do que $2 \mathrm{~cm}$ e

\begin{tabular}{lccccc}
\hline & \multicolumn{4}{c}{ Tamanho do Pólipo } \\
\cline { 2 - 6 } & \multicolumn{2}{c}{ Maior que $\mathbf{2}$ até $\mathbf{1 0} \mathbf{~ c m}$} & \multicolumn{2}{c}{ Menor do que $\mathbf{1 , 9} \mathbf{~ c m}$} \\
\cline { 2 - 6 } & $\mathbf{N}$ & $\mathbf{\%}$ & $\mathbf{N}$ & $\mathbf{\%}$ \\
\hline Perfuração & 6 & 2,9 & 0 & 0 \\
\hline
\end{tabular}

$P<0,01$. 
Tabela 4 - Síndrome pós polipectomia.

\begin{tabular}{lccccc}
\hline & \multicolumn{4}{c}{ Tamanho do Pólipo } \\
\cline { 2 - 5 } & \multicolumn{2}{c}{ Maior que 2 até $\mathbf{1 0} \mathbf{~ c m}$} & \multicolumn{2}{c}{ Menor do que $\mathbf{1 , 9} \mathbf{~ c m ~}$} \\
\cline { 2 - 5 } & $\mathbf{N}$ & $\mathbf{\%}$ & 0 & $\mathbf{N}$ \\
\hline Síndrome pós polipectomia & 6 & 2,9 & 0 \\
\hline
\end{tabular}

$P<0,01$.

Tabela 5 - Polipectomia em pólipos maiores do que $2 \mathrm{~cm}$.

\begin{tabular}{lccccc}
\hline & \multicolumn{2}{c}{ Fatiada } & \multicolumn{2}{c}{ Peça Única } \\
\cline { 2 - 5 } & $\mathbf{N}$ & $\mathbf{\%}$ & $\mathbf{N}$ & $\mathbf{\%}$ \\
\hline Sésseis & 89 & 77 & 27 & 23 \\
Pediculados & 11 & 13 & 76 & 87 \\
\hline$P<0,01$ & & &
\end{tabular}

$P<0,01$.

gigantes, quando acima de $3 \mathrm{~cm}$ de diâmetro ${ }^{7,89}$. Tivemos 203 que chamamos de grandes, com tamanho de 2 a $10 \mathrm{~cm}$ de diâmetro. Os pólipos menores do que $2 \mathrm{~cm}$ de diâmetro foram todos ressecados em peça única, porém os grandes, tiveram boa parcela deles ressecados em piecemeal, fatiados, como podemos ver na Tabela 5. Experiência semelhante é vista por outros autores ${ }^{10,11}$. Diante de pólipo pediculado gigante, fazemos o possível para ressecálo em peça única, colocando a sua base em posição tal que a negociação da alça com relação a extremidade cefálica se faça com perfeição, cuidando para evitar que fique presa apenas parte da extremidade cefálica do mesmo ou eventualmente parte do próprio cólon figuras 1,2 e 3 . Nos pólipos sésseis ou de base larga, sobretudo nos gigantes, a retirada inteira é bem mais problemática. As vezes não conseguimos se quer identificar a base com segurança. Analisamos sempre a extremidade cefálica, sua textura, consistência, a presença de úlcera e se o aspecto não for francamente favorável a adenocarcinoma, optamos pela realização de macrobiópsia com alça diatérmica, bem melhor do que avaliação de lesão vegetante vilosa por simples biópsias com pinças convencionais. Em função do aspecto vivenciado no momento deste procedimento, já que durante a macrobiópsia, muitas vezes a base fica visível e portanto se julgarmos factível a possível retirada da lesão, damos continuidade a realização da polipectomia

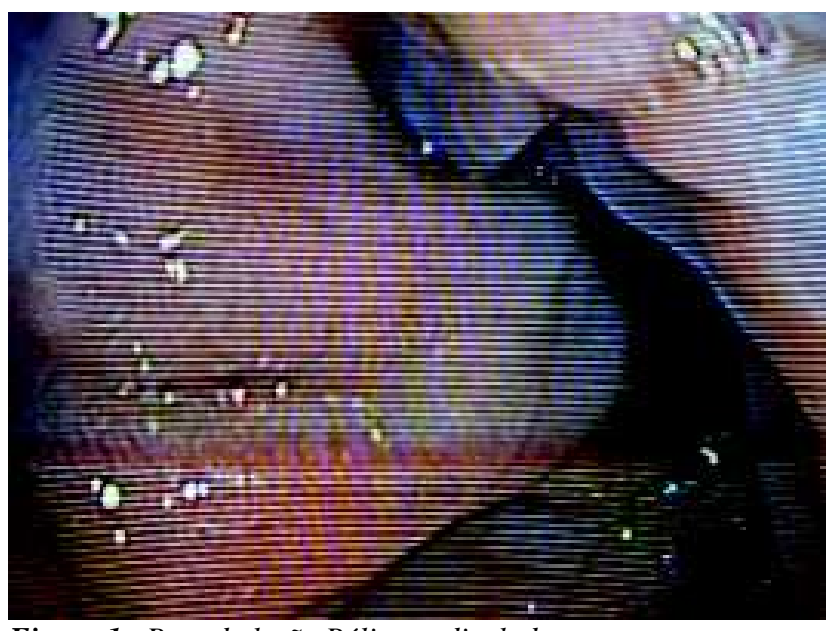

Figura 1 - Base da lesão Pólipo pediculado.

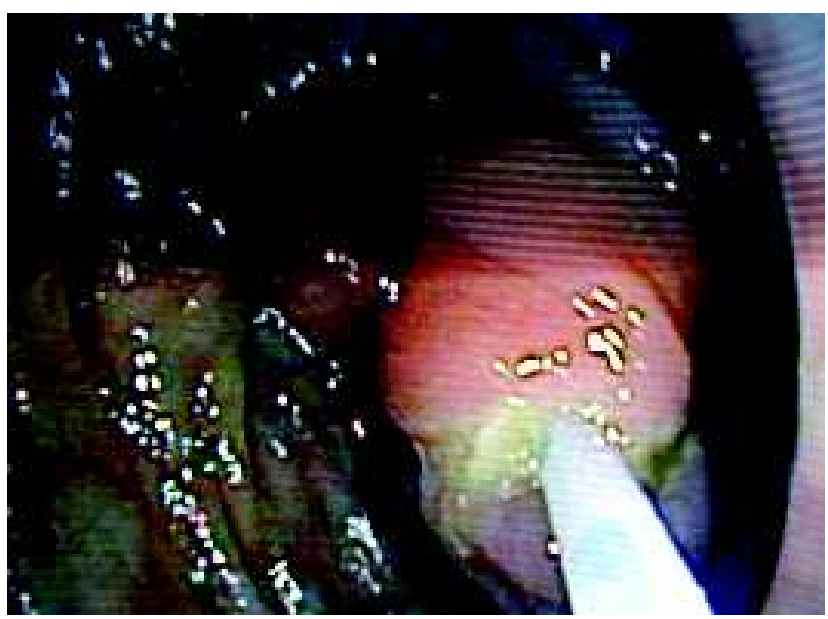

Figura 2 - Apenas o pedículo foi laçado. 


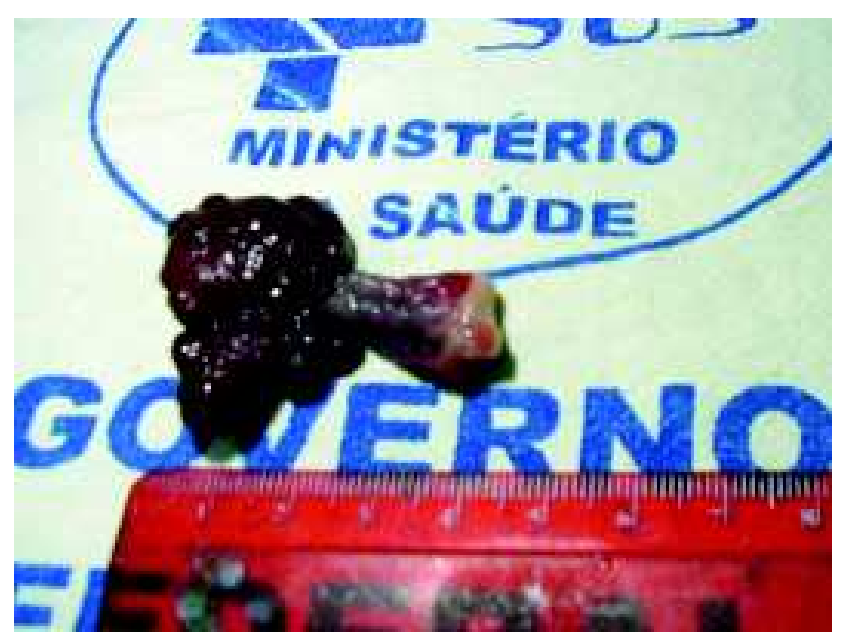

Figura 3 - Peça retirada inteira.

fatiada. Sempre complementada por técnica conjugada, isto é, a combinação com mucosectomia para retirada completa e segura das margens da lesão, como podemos ver nas figuras 4, 5, 6, 7, 8 e 9 . Se no controle endoscópico encontramos lesão residual e for benigna, tornamos a ressecar, porém agora usando a técnica da mucosectomia figuras 10, 11, 12 e 13.

A incidência de adenocarcinoma em adenoma é função do tamanho da lesão e foi encontrada em pólipos com diâmetro acima de $10 \mathrm{~mm}^{7,12,13}$. $\mathrm{Na}$ casuística deste trabalho, ocorreram em 40 pólipos $(19,7 \%)$, todos com mais de $2 \mathrm{~cm}$ em diâmetro.

As principais complicações em polipectomia com alça diatérmica são sangramento, perfuração e dor abdominal ${ }^{14,15}$ e estão associadas ao tamanho do pólipo $^{7,16,17,18,19,20}$ e são aqui demonstradas nas Tabelas 1, 2, 3 e 4. O tratamento destas complicações, em colonoscopia terapêutica, costuma ser por via endoscópica para hemorragia e conservador, não cirúrgico, para perfuração $0^{21-22}$. Todos os nossos sangramentos, com exceção de um $(0,05 \%)$ tiveram tratamento endoscópico, enquanto as perfurações, que ocorreram em ceco e cólon ascendente, tiveram todas tratamento conservador, com alta hospitalar em torno de 5 dias.

A idade dos pacientes submetidos a colonoscopia com polipectomia com alça diatérmica não teve qualquer participação nas complicações analisadas como podemos ver na tabela 6 . Não fizemos tratamento do pedículo antes de polipectomia.

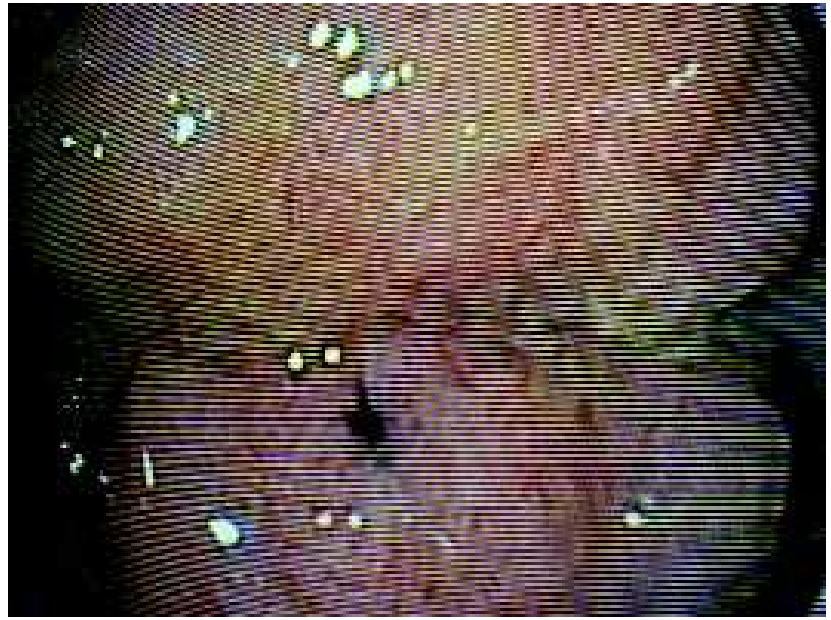

Figura 4 - Pólipo séssil.

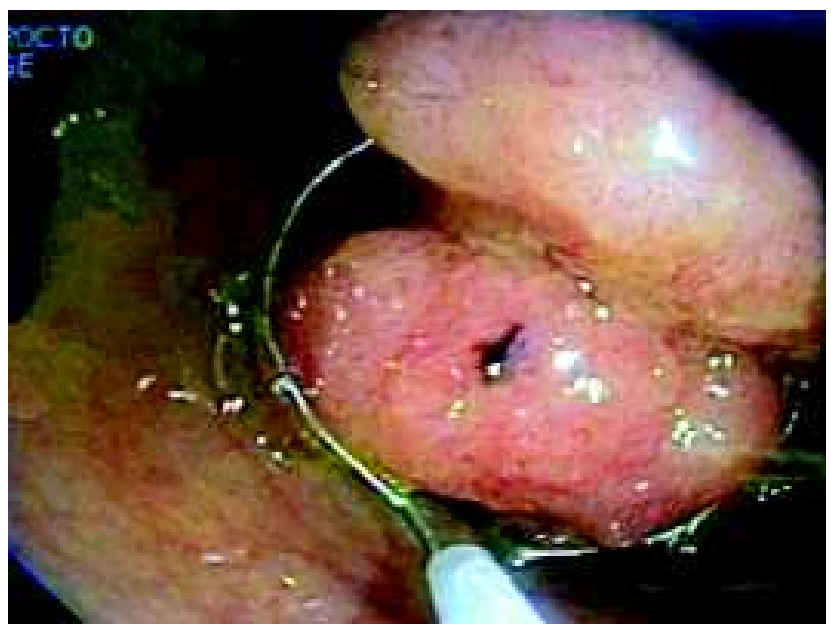

Figura 5 - Polipectomia em piecemeal.

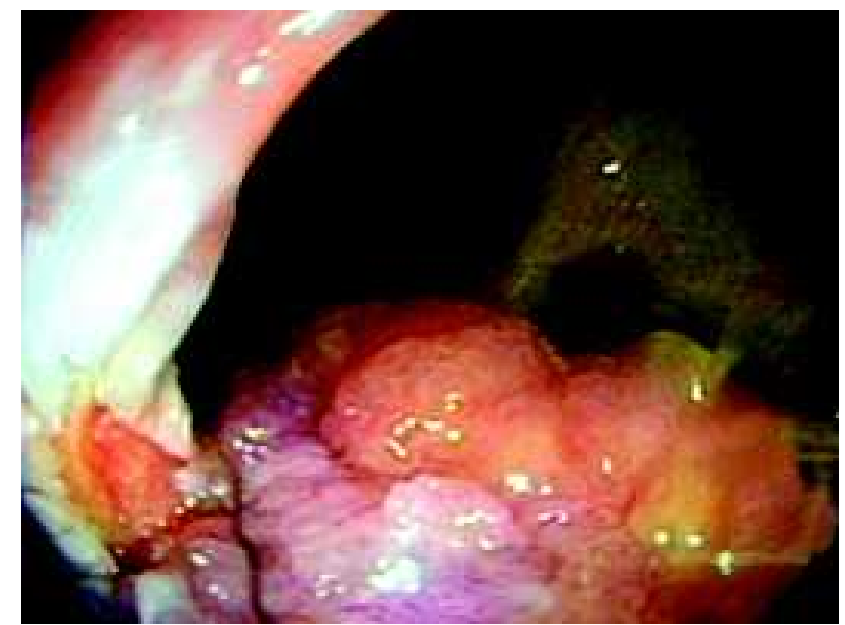

Figura 6 - Mucosectomia para retirada das margens. 


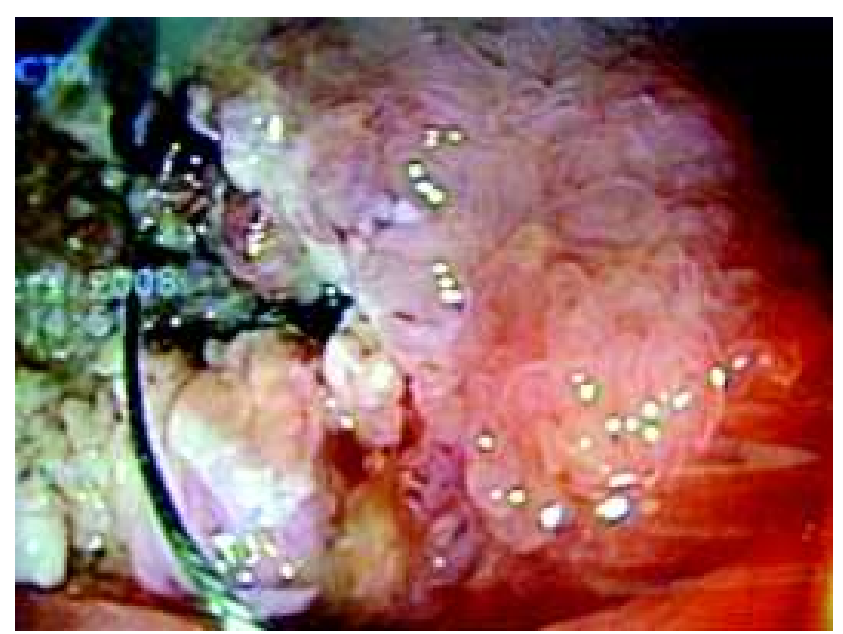

Figura 7 - Infiltração da margem para retirada segura.

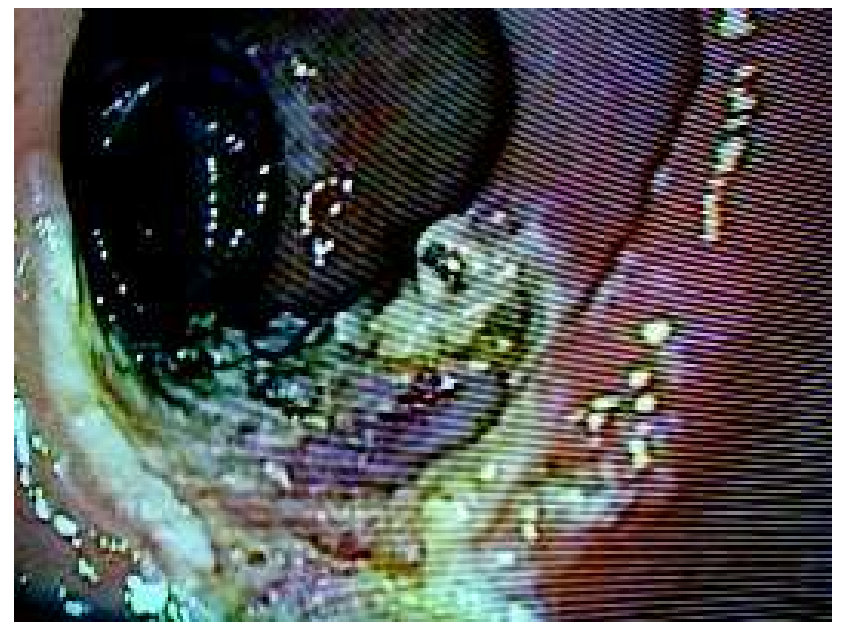

Figura 8 - Final do procedimento.

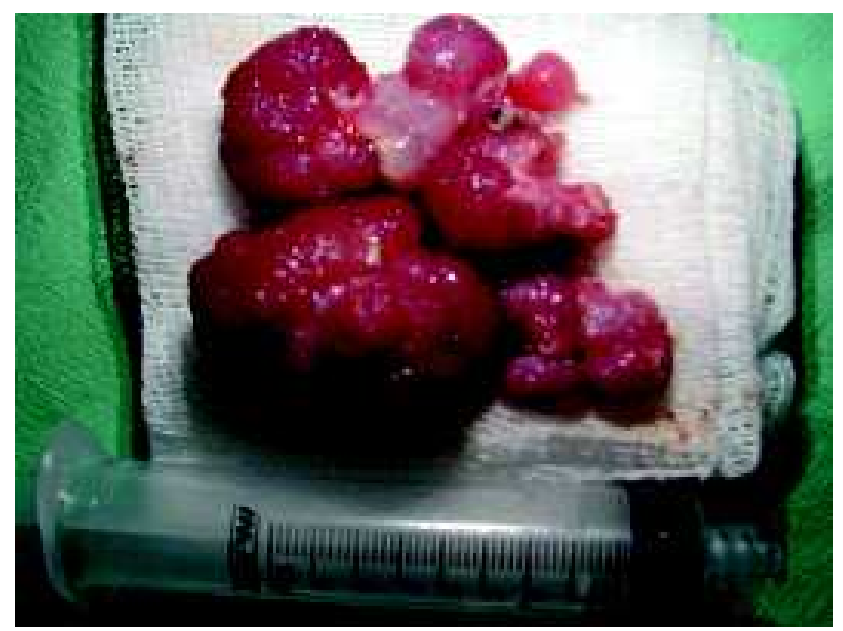

Figura 9 - Peça retirada.

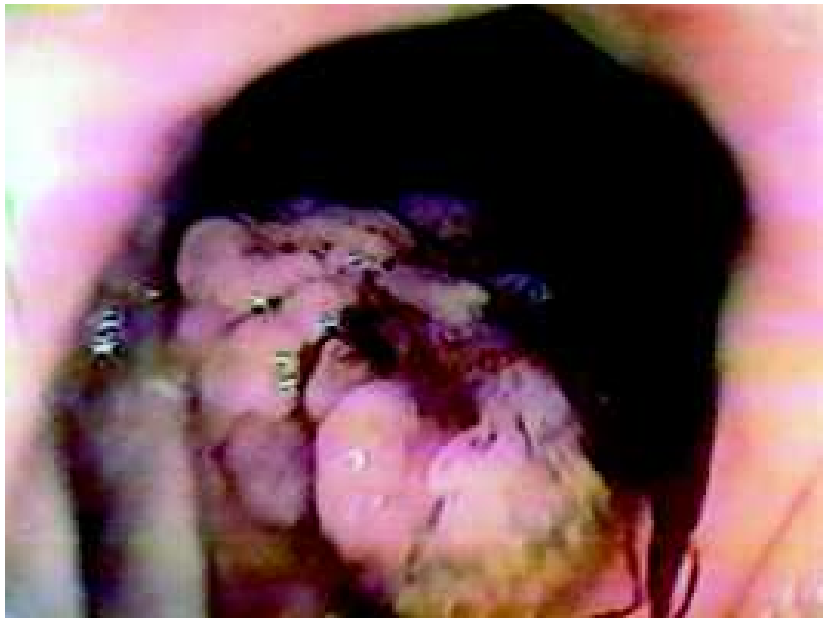

Figura 10 - Adenoma tubulo-viloso.

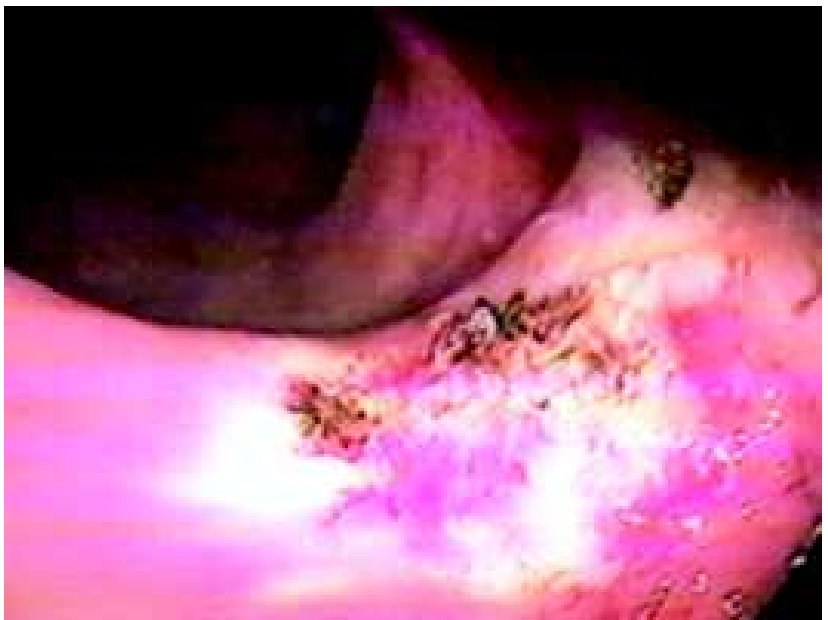

Figura 11 - Base da lesão após o término do procedimento.

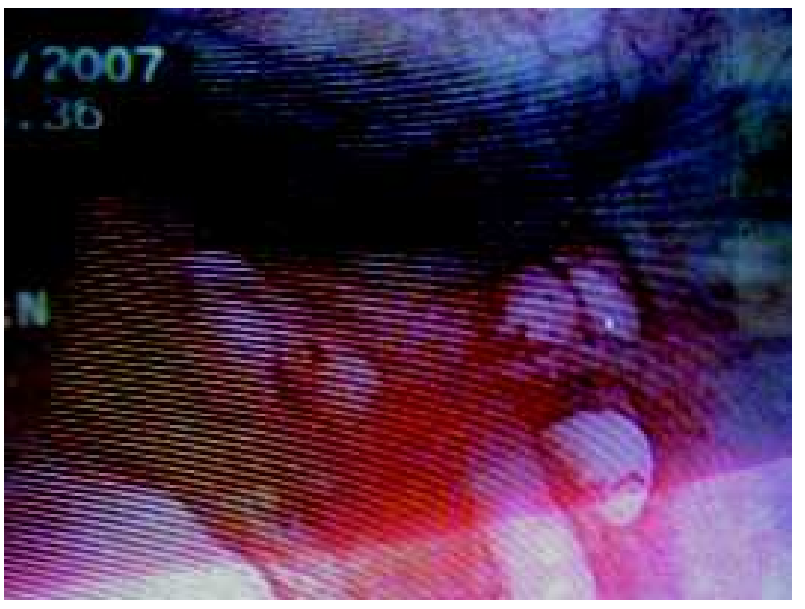

Figura 12 - Lesão residual 3 meses após: Agora mucosectomia. 


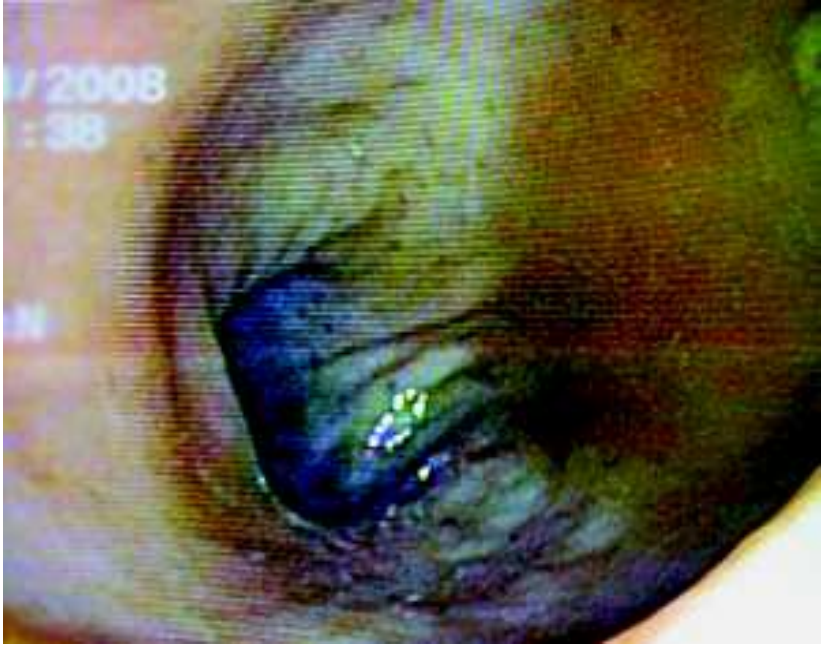

Figura 13 - Ausência de lesão residual 3 meses após.
Tabela 6 - Sangramento pós polipectomia.

\begin{tabular}{lc}
\hline & \multicolumn{1}{c}{ Idade } \\
\hline Sangramento imediato & $59,6 \pm 6,7$ \\
Sangramento tardio & $60 \pm 9,8$ \\
Sem sangramento & $63,8 \pm 16,3$ \\
\hline
\end{tabular}

$P<0,01$.

\section{CONCLUSÕES}

Polipectomia com alça diatérmica é procedimento seguro, sendo sangramento a complicação mais frequiente, relacionado ao tamanho da base do pólipo geralmente acessível a tratamento endoscópico. Perfuração vindo a seguir, tendo tratamento conservador como primeira opção.

\begin{abstract}
Purpose: The aim of this study was to evaluate adverse events from snare polypectomy. Methods: We retrospectively analysed the rate of complications of 1687 snare polypectomies carried out in 8447 colonoscopies between 2001 and 2007 at two Medical Institutions. Student $\mathbf{t}$ test was used for statistical analysis of mean and chi-square to compare absolute numbers. A significant $p$-value was defined as $<0,05$. Results: Of the 1687 colonoscopic polypectomies 203 were performed in polyps larger than $2 \mathrm{~cm}$ in diameter ( range $2-10 \mathrm{~cm}$ ). In this group $24(11,8 \%)$ had immediate bleeding, against $1(0,07 \%)$ smaller than $2 \mathrm{~cm}$ $p<0,01$. Only one, larger than $2 \mathrm{~cm}$, needed surgery to control bleeding episod $p<0,01$. Delayed bleeding occurred in $6(0,35 \%)$. None required surgery. The age group for bleeding post-polypectomy did not differ, being $59,8 \pm 6,7$ for immediate bleeding, $60 \pm 9,8$ for delayed and $63,8 \pm 16,3$ for no bleeding $p>0,05$.Post-polypectomy syndrome occurred in 6 patients $(0,35 \%)$. In polyps larger than 2 $\mathrm{cm}$, piecemeal resection was performed more often in sessile than in pedunculated ones 89/116 ( $77 \%)$ versus $11 / 87(13 \%)$ p $<0,01$. Invasive carcinoma was present in 40 adenoma larger than $2 \mathrm{~cm}(19,7 \%)$. Conclusion: Snare polypectomy is safe procedure, being bleeding the most common complication, related with polyp size mainly its base, treated most of the time endoscopically. Perforation being the next, treated too without surgery.
\end{abstract}

Key words: Colon, Colooscopy, Polyp, Polypectomy, Complication.

\section{REFERÊNCIAS}

1. Neklalson DW, Thorpe BL, Fernandez A, Tumpabura A, Boucher K, Garibotti G et al. Colonic adenoma risk in familial colorectal câncer - a study of six extended kindreds. Am J Gastroenterol 2008;103(10):2577-84.

2. Nahas SC, Imperiale AR, Araujo SE, Aisaka AA, Sobrado Junior CW, Habr-Gama A et al.Villous and tubulovillous adenomas of the colon and rectum.Results in 30 patients. Rev Hosp Clin Fac Med São Paulo 1998;53(4):162-8.

3. Brenner H, Chang-Claude J, Seiler CM, Sturmer T, Hoffmeister M. Case-control study supports extension of suveillance interval after colonoscopic polypectomy to at least $5 \mathrm{yr}$. Am J Gastroenterol 2007;102(8):1739-44.

4. Heldwein W, Dollhop M, Rosh T, Meining A, Schmidtsdorff G, Hasford J et al. The Munich polypectomy study ( MUPS): prospective analysis of complications and risk factors in 4000 colonic snare polypectomies. Endoscopy 2005;37(11):111622.

5. Jameel JK, Pillinger SH, Moncur P, Tsai HH, Duthie GS. Endoscopic mucosal resction in the management of large colorectal polyps. Colorectal Dis 2006;8(6):497-0.

6. Nahas SC, Bringel RW, Sobrado Junior CW, Nahas CS, Borba MR, Araujo SE et al. Diagnostic colonoscopy. Arq Gastroenterol 1999;36(2):72-6.

7. Consolo P, Luigiano C, Strangio G, Scaffidi MG, Giacobbe G, Di Giuseppe G et al. Efficacy, risk factors and complications of endoscopic polypectomy: ten year experience at a single center. World J Gastroenterol 2008;14(15):2364-9.

8. Dell'Abate P, Losca A, Galimberti A, Piccolo P, Soliani P, Foggi E. Endoscopic tretment of colorectal benign-appearing lesions $3 \mathrm{~cm}$ or larger: techniques and outcome. Dis Colon Rectum 2001;44(1):112-8. 
9. Overhiser AJ, Rex DK. Work and resources needed for endoscopic resection of large sessile colorectal polyps. Clin Gastroenterol Hepatol 2007;5(9):1076-9

10. Settz U, Bohnacker S, Seewald S, Thonke F, Soehendra N. Long-term results of endoscopic removal of large colorectal adenomas. Endoscopy 2003;35(8):S41-4.

11. Stergiou N, Riphaus A, Lange P, Menke D, Kockerling F, Wermann T. Endoscopic snare resection of large colonic polyps: how far can we go? Int J Colorectal Dis 2003;18(2):131-5

12. Silva EJS, Pinho PRA. Pólipos do intestino grosso e seu potencial de malignidade. GED 1991;10(2):36-0.

13. Selcuk H, Korkmaz M, Kanbay M, Tore E, Sumer H, Unal H et al. Total colonic polyp diameter: a marker for the risk of malignancy. Hepatogastrenterology 2008;55(84):936-9.

14. Mehran A, Jaffe P, Efron J, Vernava A, Liberman MA. Colonoscopy: why are general surgeons being excluded? Surg Endosc 2003;17(12):1971-3.

15. Viiala $\mathrm{CH}$, ZimmermanM, Cullen DJ, HoffmanNE. Compliction rates of colonoscopy in an Australian teaching hospital environment. Intern Med J 2003;33(8):355-9

16. Dafnis G, Ekbom A, Pahlman L, Blomqvist P. Complications of diagnostic and therapeutic colonoscopy within a defined population in Sweden. Gastrointest Endosc 2001;54(3):302-9.

17. Drobowolski S, Dobosz M, Babicki A, Glowacki J, Nalecz A. Blodd supply of colorectal polyps correlates with risk of bleeding after colonoscopic polypectomy.Gastrointest Endosc 2006;63(7);1004-9
18. Kim HS, Kim TI, Kim WH, Kim YH, Yang SH, Myung SJ et al. Risk factors for immediate postpolypectomy bleeding of the colon: a multicenter study. Am J Gastroenterol 2006;101(6):1333-41

19. Sawhney MS, Salfiti N, Nelson DB, Lederle FA, Bond JH. Risk factors for severe delayed postpolypectomy bleeding. Endoscopy 2008;40(2):115-9

20. Watabe H, Yamaji Y, Okamoto M, Kondo S, Ohta M, Ikenoue $\mathrm{T}$ et al. Risk assessment for delayed hemorrhagic complication of colonic polypectomy: polyp-related factors and patientrelated factors. Gastrointest Endosc 2006;64(1);73-8

21. Ker TS, Wasserberg N, Beart RW. Colonoscopic perforation and bleeding of the colon can be treated safely without surgery. Am Surg 2004;70(10):922-4

22. Orsoni P, Berdah S, Verrier C, Caamano A, Sastre B, Boutboul $\mathrm{R}$ et al. Colonic perforation due to colonoscopy: a retrospective study of 48 cases. Endoscopy 1997;29(3):160-4.

\section{Endereço para correspondência:}

EDSON JURADO DA SILVA

Rua Rodolfo de Souza, 105

Vila Isabel, Rio de Janeiro, RJ, Brasil

CEP: 20551-270

Tel./Fax.: (21) 2520-5115 / (21) 2293-5451

E-mail: edsonjurado@alternex.com.br 